\title{
History of research on solar energetic particle (SEP) events: the evolving paradigm
}

\author{
Edward W. Cliver ${ }^{1}$ \\ ${ }^{1}$ Space Vehicles Directorate, Air Force Research Laboratory \\ email: afrl.rvb.pa@hanscom.af.mil
}

\begin{abstract}
Forbush initiated research on solar energetic particle (SEP) events in 1946 when he reported ionization chamber observations of the first three ground level events (GLEs). The next key development was the neutron monitor observation of the GLE of 23 February 1956. Meyer, Parker and Simpson attributed this high-energy SEP event to a short time-scale process associated with a solar flare and ascribed the much longer duration of the particle event to scattering in the interplanetary medium. Thus "flare particle" acceleration became the initial paradigm for SEP acceleration at the Sun. A more fully-developed picture was presented by the Australian radio astronomers Wild, Smerd, and Weiss in 1963. They identified two distinct SEP acceleration processes in flares: (1) the first phase accelerated primarily $\sim 100 \mathrm{keV}$ electrons that gave rise to fast-drift type III emission as they streamed outward through the solar atmosphere; (2) the second phase was produced by an outward moving $\left(\sim 1000 \mathrm{~km} \mathrm{~s}^{-1}\right)$ magnetohydrodynamic shock, occurring in certain (generally larger) flares. The second phase, manifested by slow-drift metric type II emission, appeared to be required for substantial acceleration of protons and higher-energy electrons. This two-stage (or two-class) picture gained acceptance during the 1980s as composition and charge state measurements strengthened the evidence for two distinct types of particle events which were termed impulsive (attributed to flare-resident acceleration process(es)) and gradual (shock-associated). Reames championed the two-class picture and it is the commonly accepted paradigm today. A key error made in the establishment of this paradigm was revealed in the late 1990s by observations of SEP composition and charge states at higher energies $(>10 \mathrm{MeV})$ than previously available. Specifically, some large and therefore presumably "gradual" SEP events looked "impulsive" at these energies. One group of researchers attributes these unusual events to acceleration of high-energy SEPs by flares and another school favors acceleration of flare seed particles by quasi-perpendicular shocks. A revised SEP classification scheme is proposed to accommodate the new observations and to include ideas on geometry and seed particle composition recently incorporated into models of shock acceleration of SEPs.
\end{abstract}

Keywords. Sun, Solar Energetic Particles, History

\section{Introduction}

The nature of particle acceleration at the Sun, particularly at high-energies $(>30 \mathrm{MeV})$, remains a key problem in solar and solar-terrestrial physics. Here I recount the evolution of the paradigm for solar energetic particle (SEP) acceleration, beginning with the initial, implicit, picture of SEP acceleration in flares, through the emergence of the two-class (flare and shock) paradigm from 1963-1993, to challenges in the late 1990s prompted by discordant observations of ion charge states and composition at higher energies $(>10 \mathrm{MeV})$ than previously available, and to recent divergent attempts to account for these discrepancies. The history of paradigm evolution is reviewed in section 2 and in section 3 recent evidence to support shock acceleration of ions at energies $>30 \mathrm{MeV}$ 
is presented. In section 4, a revised SEP classification scheme is proposed to take into account the new SEP observations and theoretical developments on shock acceleration.

\section{Historical Review}

\subsection{Origins}

SEP research began with a question mark (actually two sets of question marks) indicating curious increases in the cosmic ray intensity observed by ionization chambers on 28 February and 7 March 1942 (Figure 1; Lange \& Forbush 1942a). Initially, Lange \& Forbush (1942b) interpreted these unusual increases (with off-scale values of $\sim 7 \%$ indicated by arrows in the figure) in terms of the magnetic effects of a ring current on galactic cosmic ray trajectories. When a subsequent event was observed on 25 July 1946, however, Forbush (Figure 2) linked it, as well as the two earlier events, to solar flares. Such increases, caused by protons with energies $\sim 1 \mathrm{GeV}$, subsequently came to be referred to as ground level events (GLEs). Forbush's 1946 paper marks the official birth of the field of SEP physics.

\subsection{The GLE of 23 February 1956 and the initial paradigm}

The next essential paper in the field was the pioneering analysis of the GLE on 23 February 1956 GLE by Meyer, Parker, \& Simpson (1956); this was the first GLE to be recorded by Simpson-developed neutron monitors. Meyer, Parker, and Simpson deduced a flare particle injection time of 20-30 minutes, an essentially "instantaneous injection" compared to the long duration ( $\sim 15$ hours) of the particle event above background. They attributed the long isotropic phase of the GLE to interplanetary scattering. Thus the initial (implicit) base level paradigm for particle acceleration at the Sun was that all particles were rapidly accelerated in the flare. Subsequently, the concepts of coronal diffusion (Reid, 1964 and Axford, 1965) and wandering interplanetary field lines (Jokipii \& Parker, 1968) were added because of the observed absence of cross-field diffusion in the interplanetary medium.

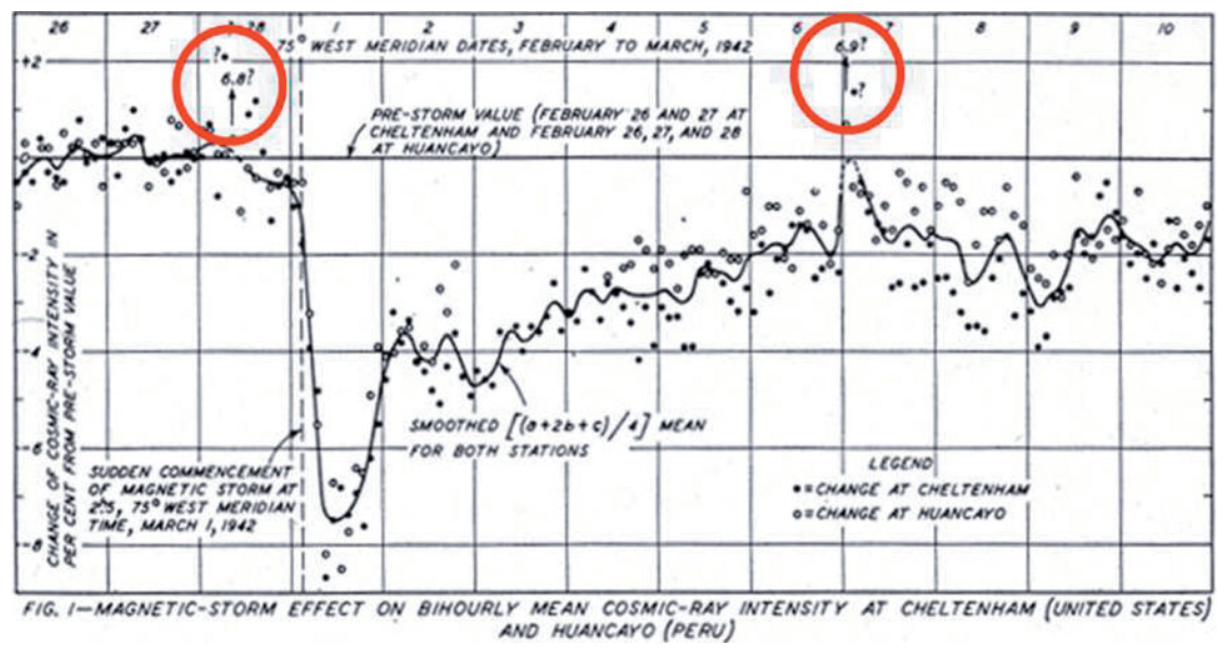

Figure 1. The first indication of solar energetic particle emission. The Cheltenham and Huancayo ionization chamber records revealed two increases (indicated by circled arrows and question marks) that Lange \& Forbush (1942a) declined to comment on at the time. 


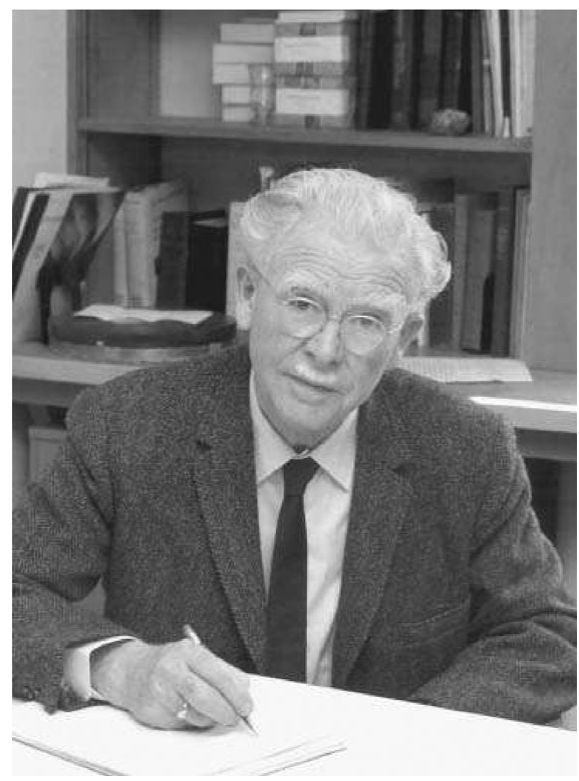

Figure 2. Scott E. Forbush (1904-1984), author of the first paper on SEPs.

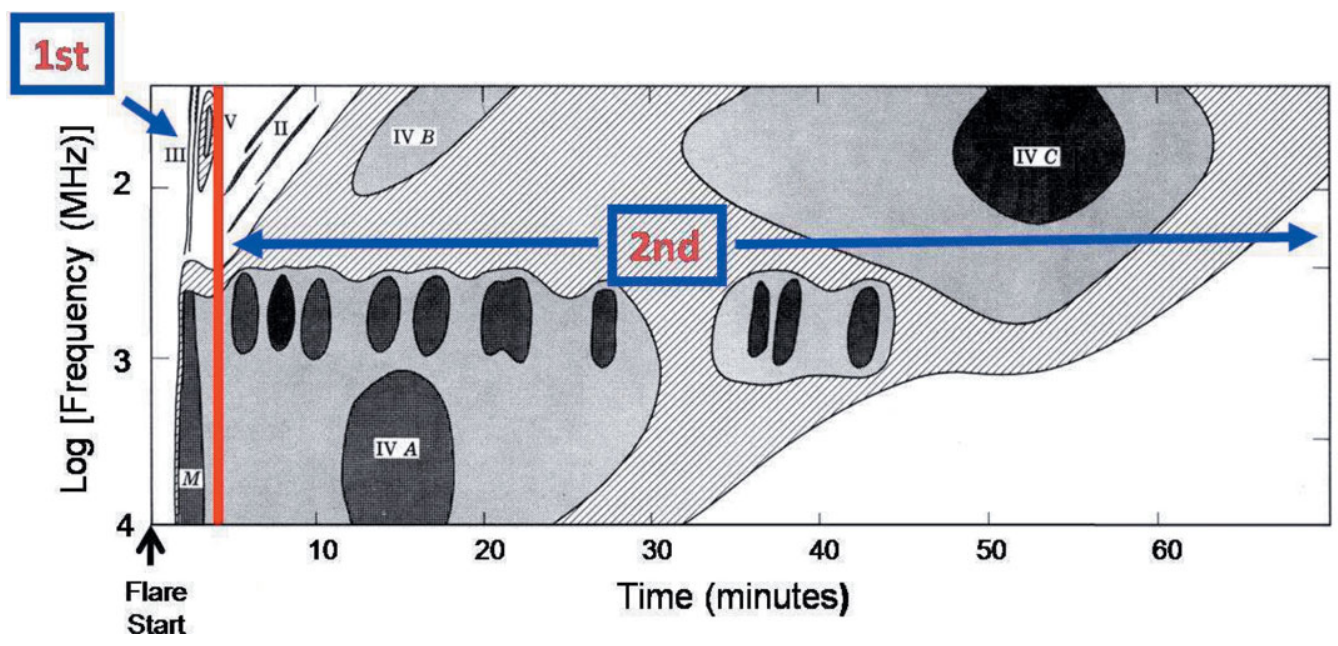

Figure 3. A fully-developed radio burst with the first (impulsive) and second (shock-associated) phases separated by the red vertical line. In the vast majority of flares, only the first phase occurs (after Wild, Smerd, and Weiss, 1963).

\subsection{The two-phase / two-class picture of SEP events}

The earliest evidence for two distinctly different particle acceleration processes on the Sun came from meter wave radio observations (Figure 3). In their Annual Reviews paper in 1963, Wild, Smerd, \& Weiss outlined a picture that is largely accepted today: SEPs are accelerated both in flares and at coronal/interplanetary shock waves. Type III bursts are the defining signature of the particle acceleration process in solar flares, while coronal shock waves are manifested by slow-drifting type II bursts. The main species accelerated during non-eruptive flares, or the impulsive (first) phase of fully-developed flares, is 
electrons of energy $\lesssim 100 \mathrm{keV}$; a SEP event with higher energy electrons and intense proton emission requires a second-phase of acceleration characterized by a shock. $\dagger$

Early SEP observations from satellites provided support for this picture. Lin (1970) distinguished between "mixed" SEP events for which both protons and relativistic electrons were observed and low-energy $(\sim 40 \mathrm{keV})$ "pure electron" events. The mixed events were generally preceded by metric type II solar radio bursts while the pure electron events were characteristically associated with metric type III emission. In 1978 Kahler, Hildner, \& Van Hollebeke showed that prompt solar proton events were strongly associated with the then recently discovered coronal mass ejections (CMEs). The standard interpretation of this observation is that fast CMEs drive shock waves that produce type II radio emission and accelerate protons and high-energy electrons.

The underpinnings of the modern two-class picture, which involves $\mathrm{Z} \geqslant 2$ ions as well as protons and electrons, can be traced to a remarkable series of papers from 1984-1986. In 1984 , Klecker et al. were the first to show that ${ }^{3}$ He-rich SEP events and large "normal" SEP events had quite different Fe charge state distributions. While large SEP events typically had low $(\sim 14)$ Fe charge states, somewhat above that of Fe in the solar wind, the Fe ions in the small ${ }^{3}$ He-rich events (discovered in 1970 by Hsieh \& Simpson) had Fe charge states $\sim 19$, suggesting that these events were processed in the high-temperature flare. In a key paper in 1985, Reames, von Rosenvinge, \& Lin discovered that ${ }^{3} \mathrm{He}$ ions were an emission of the impulsive phase of flares by associating them with low-energy electrons, the principal particle species of impulsive flares.

Mason et al. (1986) showed that heavier ions in ${ }^{3} \mathrm{He}$-rich events had a distinctive abundance pattern, with Fe enriched by a factor of $\sim 10$ over that observed in large SEP events. The two types of SEP events inferred by Wild, Smerd, \& Weiss (1963) on the basis of solar radio observations and electron and proton spectra were exhibiting distinctions between themselves over a broader range of SEP species and characteristics.

Cane, McGuire, \& von Rosenvinge (1986) separated SEP events into two classes on the basis of the durations of their associated flares. Their "impulsive" SEP events had soft $\mathrm{X}$-ray durations $\leqslant 1$ hour, high electron-to-proton $(\mathrm{e} / \mathrm{p})$ ratios, and were well-connected, i.e., had associated flares clustered near the $\sim$ W60 footpoint of the nominal Parker spiral field line connecting the Sun to Earth. The "gradual" SEP events had flare durations $>1$ hour, higher proton intensities, and could originate anywhere on the solar disk. What was not appreciated at the time, however, was that many of the impulsive events of Cane, McGuire, and von Rosenvinge were far more energetic than the ${ }^{3} \mathrm{He}$-rich events of Reames, von Rosenvinge, \& Lin (1985).

One other paper from 1984-1986 requires mention here. Breneman \& Stone (1985) reported that "the ionic charge-to-mass ratio $(\mathrm{Q} / \mathrm{M})$ is the principal organizing factor for the fractionation of ... SEPs by acceleration and propagation processes and for flare-toflare variability". It would take $\sim 20$ years before this striking result found an explanation within the two-class picture.

In the meantime, the accumulating evidence made a compelling and correct case for two fundamentally different types of SEP events (or acceleration processes on the Sun): (1) "Impulsive" events linked to the flare acceleration process, presumably a resonant wave-particle interaction that resulted in preferential acceleration of electrons, ${ }^{3} \mathrm{He}$, and high-Z ions; and (2) large "gradual" events attributed to shock acceleration of solar wind

$\dagger$ In 1983, Forrest \& Chupp presented evidence from gamma-ray observations that protons and high-energy electrons are also accelerated in the impulsive phase of high energy flares. Subsequently, Ramaty, Murphy, \& Dermer (1987) interpreted a delayed "pion-rich" phase of emission in the 3 June 1982 flare in terms of a second phase acceleration process, echoing Wild, Smerd, \& Weiss (1963). 
Table 1. Two-class Paradigm for SEP Events

\begin{tabular}{ccc}
\hline \hline & IMPULSIVE & GRADUAL \\
\cline { 2 - 3 } & & \\
Particles: & Electron-Rich & Proton-Rich \\
${ }^{3} \mathrm{He} /{ }^{4} \mathrm{He}$ & $\sim 1$ & $\sim 0.0005$ \\
$\mathrm{Fe} / \mathrm{O}$ & $\sim 1$ & $\sim 0.1$ \\
$\mathrm{H} / \mathrm{He}$ & $\sim 10$ & $\sim 100$ \\
$\mathrm{Q}_{F e}$ & $\sim 20$ & $\sim 14$ \\
Duration & Hours & Days \\
Longitude Cone & $<30^{\circ}$ & $\sim 180^{\circ}$ \\
Radio Type & III,V(II) & II,IV \\
X-Rays & Impulsive & Gradual \\
Coronograph & - & CME \\
Solar Wind & - & IP Shock \\
Events/Year & $\sim 1000$ & $\sim 10$ \\
\hline
\end{tabular}

particles. The classification scheme is shown in its iconic two-column form in Table 1. Don Reames $(1993,1999)$ was the primary author of this picture and a driving force in SEP physics for two decades, effectively taking it from a pre-paradigmatic state (in the sense that before $\sim 1990$ there was no consensus paradigm or generally-accepted paradigm) to one with a well-defined framework.

As hinted at above, there was an inherent flaw in the scheme, revealed by the fact that "type II" appears in the 'Radio Type' row of both columns in Table 1, when one would think that SEP acceleration by large-scale shocks would only play a role in gradual events. The impulsive class combined two quite different types of events - the ${ }^{3} \mathrm{He}-\mathrm{rich}$ events of Reames, von Rosenvinge, \& Lin (1985) which characteristically lacked CMEs, type II bursts, and detectable protons (Kahler et al., 1985), and the much more energetic impulsive events from Cane, McGuire, \& von Rosenvinge (1986) that were accompanied by each of these phenomena. All that the two types of events had in common was the short duration of the soft X-ray flares. The Cane et al. SEP events were selected on the basis of detection of $>3 \mathrm{MeV}$ electrons in comparison with the $\lesssim 100 \mathrm{keV}$ electrons typically observed for ${ }^{3} \mathrm{He}-\mathrm{rich}$ SEP events.

In an attempt to separate the energetic impulsive SEP events of Cane et al. (1986) from the ${ }^{3}$ He-rich events of Reames, von Rosenvinge, \& Lin (1985), Cliver (1996; see also Kallenrode, Cliver, \& Wibberenz, 1992) proposed a modification of the two-class picture in which these two types of events were represented by separate columns. The standard two-class paradigm (Table 1) was well-entrenched by 1996, however, and the worry that the higher-energy events of Cane et al. were not qualitatively the same as the ${ }^{3} \mathrm{He}$-rich events was insufficient cause to modify the picture. The case for revision would have to await additional rationale from SEP observations.

\subsection{Recent challenge to the two-class paradigm}

The inherent flaw in the two-class picture was revealed in 1999 with the publication of ACE observations of several large events observed early in cycle 23. Cohen et al. (1999) found that events on 6 November 1997, 2 May 1998, 6 May 1998, and 14 November 1998, had elemental compositions at $>10 \mathrm{MeV}$ that looked remarkably like those tabulated by Reames for impulsive events at lower energies (Figure 4a). Since all large events were assumed to be gradual, this posed a problem - a problem that was exacerbated when 
(a)
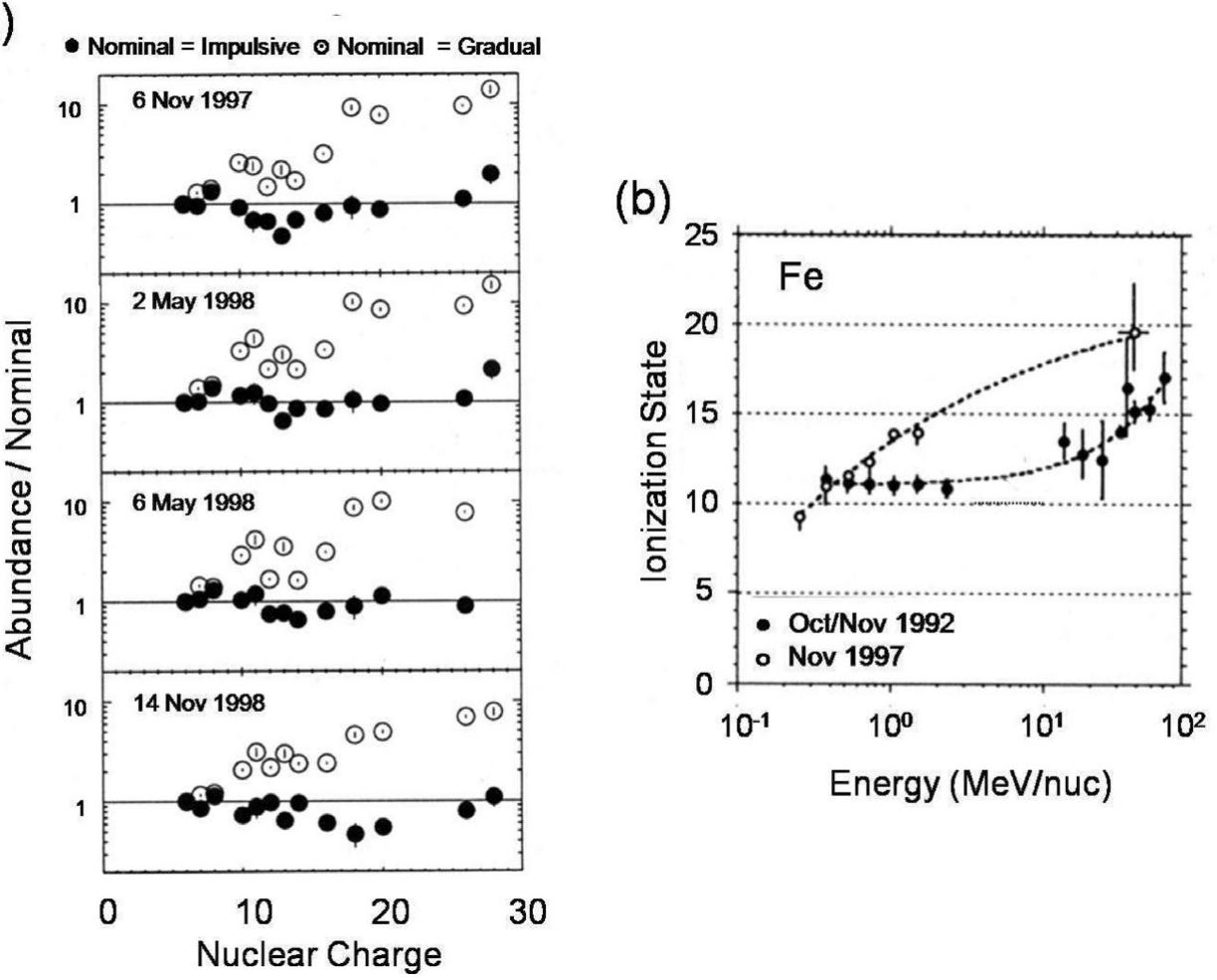

Figure 4. (a) Four of the early large ACE events had impulsive compositions at $>10 \mathrm{MeV}$ energies (from Cohen et al., 1999) and (b) at least two of these (one shown) had high Fe charge states (from Mazur et al., 1999).

SAMPEX measurements of charge states at $\sim 40 \mathrm{MeV}$ using the geomagnetic cutoff technique (Mazur et al., 1999) revealed that two of the events (6 November 1997, 14 November 1998) had high Fe charge states (6 November shown in Figure 4b).

Attempts to accommodate the new observations in terms of the two-class picture of SEP events took two disparate paths. The first, after Cane and colleagues (Cane et al., 2002, 2003, 2006), argued that the "impulsive" properties observed in the large "gradual" events were due to flare domination of the gradual events at high $(>25 \mathrm{MeV})$ energies. Alternatively, Tylka and colleagues (Tylka et al., 2005, 2006; Tylka \& Lee, 2006) accounted for the enhanced $\mathrm{Fe} / \mathrm{O}$ ratios and charge states in the events of Cohen et al. in terms of quasi-perpendicular shocks operating on a flare suprathermal seed population.

Cane et al. (2002) pointed out that strong type III bursts observed with the WAVES instrument on the Wind spacecraft provided a direct link between the flare and the observation of protons at $1 \mathrm{AU}$. [This argument is problematic because there is evidence that some type IIIs may actually originate in shocks.] For the shock-based solution, the key phenomenon is a coronal shock wave manifested by a type II burst in the decametrichectometric (DH) range (Gopalswamy et al., 2002; Cliver, Kahler, \& Reames, 2004).

Cane et al. (2006) noted that events with high Fe/O ratios came from well-connected solar longitudes. This is consistent with a flare source since flares are generally thought to have a smaller "cone of SEP emission" than shocks. These authors linked well-connected events with low Fe/O ratios to strong interplanetary shocks and/or more complex SEP time-intensity profiles. 


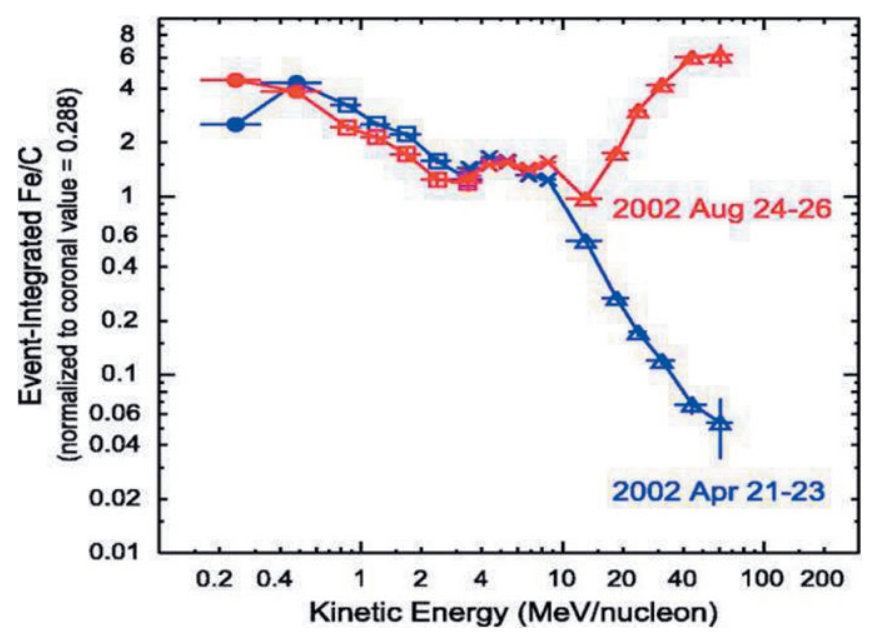

Figure 5. Examples of the two limiting cases of Fe/O variation with energy in large SEP events (from Tylka et al., 2006).

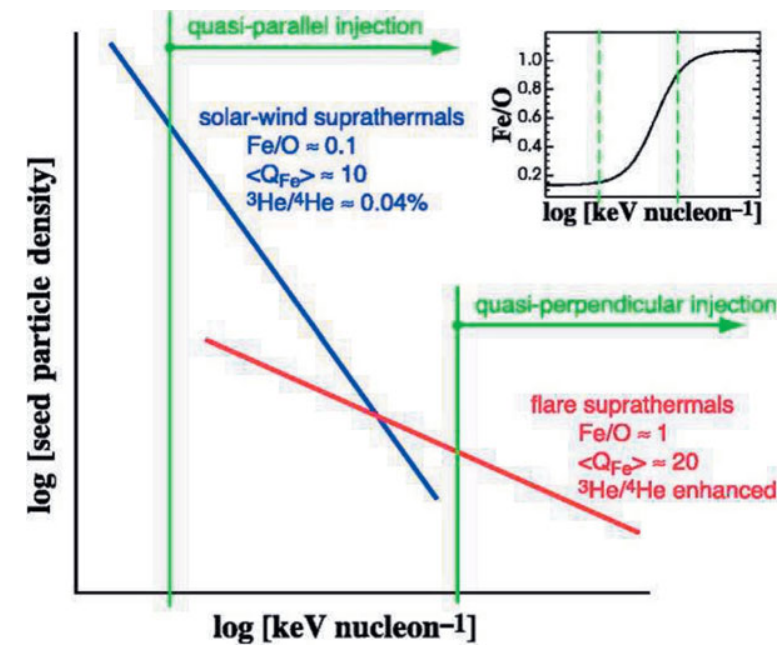

Figure 6. Schematic representation of the seed populations for shock-accelerated SEPs. Because of their higher energies, flare suprathermals (STs) are more accessible to quasi-perpendicular shocks. The inset shows how the $\mathrm{Fe} / \mathrm{O}$ ratio in the seed population varies with energy (from Tylka et al., 2005).

Tylka et al. $(2005,2006)$ examined the variation of the $\mathrm{Fe} / \mathrm{O}$ ratio in SEP events with ion energy and noted two extreme possibilities for which $\mathrm{Fe} / \mathrm{O}$ at $\sim 40 \mathrm{MeV}$ varied by approximately two orders of magnitude (Figure 5). They noted that similar qualitative behavior was observed in situ for shock-associated SEP events observed at 1 AU - an example of a Universal Heliophysical Process - and that in that case the difference could be ascribed to shock geometry, with events with increasing $\mathrm{Fe} / \mathrm{O}$ ratio with energy being linked to quasi-perpendicular shocks. They also noted that quasi-parallel shocks can operate on relatively low energy seed particles such as coronal or solar wind suprathermals while quasi-perpendicular shocks have a higher injection energy requirement, favoring flare suprathermals. Thus, given the seed particle populations shown schematically in Figure 6, quasi-perpendicular shocks are more likely to produce SEP events with 
enhanced $\mathrm{Fe} / \mathrm{O}$ while SEP events from quasi-parallel shocks will tend to have low $\mathrm{Fe} / \mathrm{O}$ ratios. The various combinations of shock geometry and seed particle population variability account for the wide variation observed in SEP composition and charge states for large events. With their shock formulation, Tylka \& Lee (2006) were able to reproduce the organization of SEP elemental abundances by charge/mass ratio discovered by Breneman \& Stone (1985) and provide the first theoretical explanation for this effect.

\section{Evidence in support of shock acceleration of $>30 \mathrm{MeV}$ SEPs}

In this section I summarize recent work by myself and colleagues relating to the challenge posed by the observations of Cohen et al. (1999) and Mazur et al. (1999) and the differing explanations suggested by the Cane and Tylka camps.

\subsection{Associations of large SEP events with low-frequency radio bursts}

While metric type II bursts are a quasi-necessary condition for a large SEP event, they are not sufficient. The fact that many metric type IIs are not followed by SEPs at Earth raises a question about the role of type IIs in the cases where there is a linkage. Is there something special about the type IIs linked to high-energy (>20 MeV) SEP events at Earth? To address this question, Cliver, Kahler, \& Reames (2004) considered DH type IIs (those observed between $14-1 \mathrm{MHz}$ by the Wind/WAVES instrument). They found that from July 1996 - June 2001 metric type IIs with a DH counterpart were $\sim 3.5$ times more likely to have $>20 \mathrm{MeV}$ SEP association. This suggests simply that stronger shocks, i.e., those capable of persisting to $\sim 3$ solar radii, are more likely to produce a detectable $>20 \mathrm{MeV}$ SEP event at $1 \mathrm{AU}$. This correlation of SEP size with an indicator of shock strength provides general support for the shock scenario for large SEP events.

More recently, Cliver \& Ling (2009) started with a sample of large favorably-located $\sim 1 \mathrm{MHz}(\sim 7 \mathrm{Rs})$ type III bursts from 1997-2004 and examined their association with large $[\geqslant 1$ proton flux unit $(\mathrm{pfu})]>30 \mathrm{MeV}$ proton events. They found that to first order such bursts were associated with large $>30 \mathrm{MeV}$ SEP events only when they were accompanied by DH type II bursts. This indicates that strong shocks rather than strong flares are required for $>30 \mathrm{MeV}$ SEP acceleration at the Sun.

\subsection{Flare time scale and shock geometry}

Flare time-scale is an important parameter in the current two-class picture, with all gradual SEP events having flares with long ( $>1 \mathrm{hr}$ ) soft X-ray durations. Yet well-known intense events such as 3 June 1982 and 6 November 1997 were associated with short duration ( $<1$ hour) flares. Is there a relationship between flare duration, shock geometry and SEP composition? We suggest the following link: Short flare time scale indicates that the eruption originates on compact spatial scales. Upon eruption, the CME undergoes rapid lateral expansion, driving a shock tangential to the solar surface with shock normal perpendicular to the radial magnetic field, i.e., a quasi-perpendicular shock, resulting in enhanced $\mathrm{Fe} / \mathrm{O}$ and e/p ratios. More gradual eruptions originate on larger spatial scales with a slower rate of energy release and the resultant lateral expansion is less violent, suggesting a reduced role for quasi-perpendicular shocks. In the context of Tylka's shock model, it appears that the short duration flares are preferentially linked to quasi-perpendicular shocks. The cartoon in Figure 7 shows where quasi-parallel and quasi-perpendicular shock acceleration of SEPs might occur in relation to a CME. In this scenario, events like those observed by ACE in 1997-1998 (Cohen et al., 1999) are explained in terms of quasi-perpendicular acceleration and, indeed, in the 6 November 


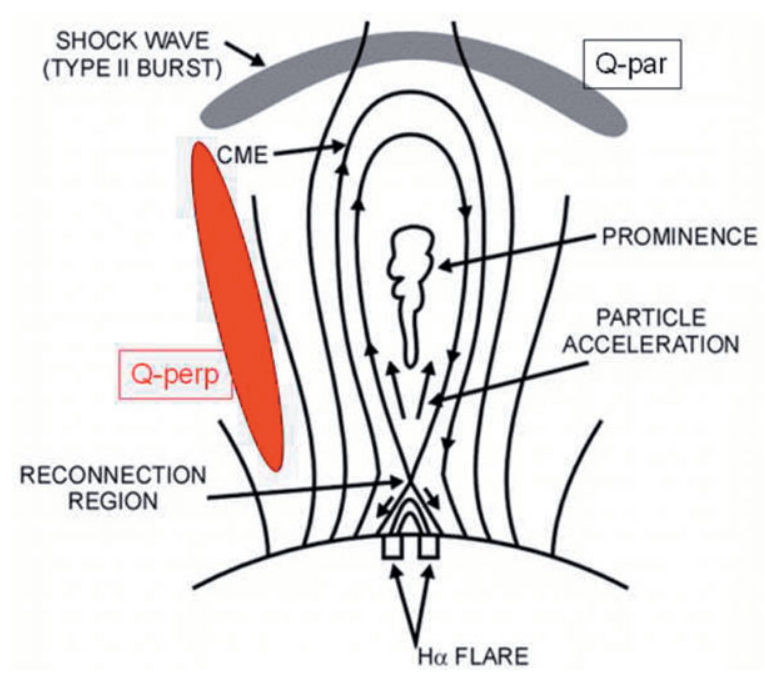

Figure 7. Schematic showing where quasi-parallel and quasi-perpendicular shock acceleration might occur in a solar eruption. In the standard CSHKP picture for eruptive flares (see Hudson \& Cliver, 2001), reconnection in the wake of the CME gives rise to a two-ribbon flare. If these flare particles escape the CME, they could become seed particles for the shock.

1997 event, there is evidence (Zhang et al., 2001) for lateral expansion of a CME that could drive such a shock. The incorporation of flare time scale as a parameter in the shock events finds support from the study of Nitta, Cliver, \& Tylka (2003) that linked certain events with high Fe/O ratios, including the 6 November 1997 event, to "explosive" eruptions on the Sun, and others with low Fe/O ratios to less abrupt mass ejections.

Cliver (2008b) provides more general evidence that time scale is a crude organizer of $\mathrm{Fe} / \mathrm{O}$ in large SEP events, with a tendency for shorter flares to be associated with $\mathrm{SEP}$ events with higher $\mathrm{Fe} / \mathrm{O}$, as well as e/p, ratios. The tendency for enhanced e/p ratios is consistent with the shock-based picture of Tylka and colleagues because on both theoretical (Lee, 2005) and observational (Tsurutani \& Lin, 1985) grounds, we would expect quasi-perpendicular shocks (preferentially arising in short-duration flares) to be efficient accelerators of electrons in comparison with quasi-parallel shocks.

\section{A proposed revision of the two-class taxonomy of SEP events}

The observations of impulsive characteristics in certain large SEP events indicate the need for a modification of the standard two class picture (Cliver, 2008a). Following the lead of Tylka and his co-workers, we propose that the current gradual class be divided into two subclasses on the basis of shock geometry (and seed particles): quasi-perpendicular shocks (operating on flare seed particles) and quasi-parallel shocks (operating on coronal / solar wind suprathermals) (Table 2). This revision reflects the differences between the ${ }^{3}$ He-rich events [now Flare] of Reames, von Rosenvinge, \& Lin (1985) and the energetic impulsive events [Quasi-Perp] of Cane, McGuire, \& von Rosenvinge (1986) which were combined in the original two-class picture (Reames, 1993). As a semantic change, we replace the main heading terms "Impulsive" and "Gradual" with "Flare" and "Shock", respectively. It is important to note that the two shock subclasses are extremes of a continuum. The notion of two basic types of SEP events remains intact. SEPs are accelerated in flares and at coronal shock waves. The supporting evidence for altering the classification scheme in this manner (see also Cliver, 2008b) is as follows: (1) The 
Table 2. Revised SEP Event Classification ${ }^{a}$

\begin{tabular}{cccc}
\hline \hline & $\underline{\text { Flare }}$ & \multicolumn{2}{c}{ Shock } \\
& & Quasi-Perp & $\underline{\text { Quasi-Par }}$ \\
\hline H Upper Limit $^{b}$ & $\sim 3 \mathrm{pr}$ & $\sim 10^{3} \mathrm{pr}$ & $\sim 10^{4} \mathrm{pr}$ \\
$\mathrm{e} / \mathrm{p}^{b}$ & $\sim 10^{2}-10^{4}$ & $\sim 100$ & $\sim 50$ \\
${ }^{3} \mathrm{He} /{ }^{4} \mathrm{He}^{c}$ & $\sim 10^{3}-10^{4}$ & $\sim 10^{1}-10^{2}$ & $\sim 1$ \\
$\mathrm{Fe} / \mathrm{O}^{d}$ & $\sim 8$ & $\sim 3$ & $<1$ \\
$\mathrm{Z}(>50) / \mathrm{O}^{e}$ & $\sim 10^{2}-10^{3}$ & $\sim 10^{-1}-10^{1}$ & $\sim 10^{-1}-10^{1}$ \\
Ion Spectra & - & Power-law & Exp. Rollover \\
QFe $^{g}$ & $\sim 20$ & $\sim 20$ & $\sim 11$ \\
SEP Duration & $<1-20 \mathrm{hr}$ & $\sim 1-3$ days & $\sim 1-3$ days \\
Longitude Cone $^{h}$ & $<30-70^{\circ}$ & $\sim 100^{\circ}$ & $\sim 180^{\circ}$ \\
Seed Particles & $\mathrm{N} / \mathrm{A}$ & Flare STs & Coronal STs \\
Radio Type & III & II & II \\
X-ray Duration & $10-60 \mathrm{~min}$ & $\sim 1 \mathrm{hr}$ & $>1$ hr \\
Coronagraph $^{j}$ & $*$ & CME & CME \\
Solar Wind $^{*}$ & - & IP Shock & IP Shock \\
\hline
\end{tabular}

Notes to Table 2:

${ }^{a}$ See Cliver (1996) for an early attempt to accommodate the quasi-perpendicular shock events.

${ }^{b}$ Cliver \& Ling (2007), Cliver (2008b); >10 MeV protons and $0.5 \mathrm{MeV}$ electrons.

${ }^{c}$ Relative to solar wind at $\sim 1 \mathrm{MeV} /$ nuc. The "problem" large SEP events on 6 November 1997, 2 May 1998, and 6 May 1998 had enhanced ${ }^{3} \mathrm{He} /{ }^{4} \mathrm{He}$ ratios.

${ }^{d}$ Relative to corona at $5-12 \mathrm{MeV} /$ nuc. For the Flare class, the energy range is $5-12 \mathrm{MeV} / \mathrm{nuc}$

(Reames, 1999). For the Shock class, the energy range is 30-40 MeV/nuc (Tylka et al., 2005).

${ }^{e}$ Relative to corona at $5-12 \mathrm{MeV} /$ nuc; Reames and $\mathrm{Ng}$ (2004).

${ }^{f}$ For a study of ion spectra in Flare events, see Mason et al. (2002). Spectral shapes for Shock events are for 3-100 MeV/nuc (Tylka et al., 2005).

${ }^{g}$ Flare (<1 MeV/nuc; Klecker et al., 1984); Shock ( 40 MeV/nuc; Mazur et al., 1999).

${ }^{h}$ Lin (1970); Reames, Stone, \& Kallenrode (1991); Kallenrode, Cliver, \& Wibberenz (1992).

${ }^{i}$ Defining radio type in low frequency range from $14-1 \mathrm{MHz}$.

${ }^{j}$ The larger flare events can have associated CMEs (Kahler, Reames, \& Sheeley, 2001).

similarity between $\mathrm{Fe} / \mathrm{O}$ variation with energy in quasi-perpendicular solar and interplanetary shock events (Tylka et al., 2005); 2) New insight on the $\sim 20$ year old puzzle of the Breneman \& Stone (1985) Q/M fractionation effect provided by Tylka \& Lee (2006); (3) Incorporation of flare-time scale into the division of the shock class into two subtypes (it is always a positive sign when a new view of nature encompasses key aspects of the superseded picture); (4) Mounting evidence that strong shocks, rather than strong flares, are a requirement for large $>25 \mathrm{MeV}$ SEP events (e.g., Cliver \& Ling, 2009); and (5) Occam's razor - if the middle column in Table 2 were attributed to a different type of flare process, the strong (DH) shocks observed in these events would be extraneous.

Since this field began with question marks (Figure 1), it seems appropriate to end this review with some more. Much remains to be done, e.g., detailed mechanisms for both the flare and shock SEP acceleration processes. Sticking points, observations that do not fit neatly into the working hypothesis of Table 2, hold potential for insight and revision. To mention two: (1) the unusual long-duration pion-rich gamma-ray events observed by GRO Comptel (Kanbach, 1993; Ryan, 2000; see Cliver 2006); and (2) flattening spectra for high-energy electron events associated with short-duration flares (Moses et al., 1989). It remains to be seen how or if these phenomena will be incorporated into Table 2. 


\section{Acknowledgements}

I thank N. Gopalswamy, D. Webb, and K. Shibata for organizing this stimulating meeting and asking me to speak on this topic. Figures 5 and 6 reproduced by permission of the AAS.

\section{References}

Axford, W. I. 1965, Planet. Space Sci., 13, 1301

Breneman, H. H. \& Stone, E. C. 1985, ApJ (Lett.), 299, L57

Cane, H. V., McGuire, \& von Rosenvinge, T. T. 1986, ApJ, 301, 448

Cane, H. V., Erickson, W. C., \& Prestage, N. P. 2002, J. Geophys. Res., 107(A10), CiteID 1315

Cane, H. V., von Rosenvinge, T. T., et al. 2003, Geophys. Res. Lett., 30(12), CiteID 8017

Cane, H. V., Mewaldt, R. A., et al. 2006, J. Geophys. Res., 111(A6), CiteID A06S90

Cliver, E. W. 1996, in High Energy Solar Physics, eds., R. Ramaty, N. Mandzhavidze, \& X.-M. Hua, AIP, Woodbury, NY, vol. 374, p. 45

Cliver, E. W., Kahler, S. W., \& Reames, D. V. 2004, ApJ, 605, 902

Cliver, E. W. 2006, ApJ, 639, 1206

Cliver, E. W. \& Ling, A. G. 2007, ApJ, 658, 1349

Cliver, E. W. \& Ling, A. G. 2009, ApJ, 690, 598

Cliver, E. W. 2008a, in Particle Acceleration and Transport in the Heliosphere and Beyond, eds., G. Li, Q. Hu, O. Verkhoglyadova, G. Zank, R. Lin, \& J. Luhmann, AIP, Melville, NY, vol. 1039 , p. 190

Cliver, E. W. 2008b, Central European Astrophys. Bull. (in press)

Cohen, C. M. S., et al. 1999, Geophys. Res. Lett., 26, 2697

Forbush, S. E. 1946, Phys. Rev., 70, 771

Forrest, D. J. \& Chupp, E. L. 1983, Nature, 305, 291

Gopalswamy, N., et al. 2002, ApJ (Lett.), 572, L103

Hsieh, K. C. \& Simpson, J. A. 1970, ApJ (Lett.), 162, L191

Hudson, H. S. \& Cliver, E. W. 2001, J. Geophys. Res., 106, 25199

Jokipii, J. R. \& Parker, E. N. 1968, Phys. Rev. Lett., 21, 44

Kahler, S. W., Hildner, E., \& Van Hollebeke, M. A. I. 1978, Solar Phys., 57, 429

Kahler, S., Reames, D. V., et al. 1985, ApJ, 290, 742

Kalher, S. W., Reames, D. V., \& Sheeley, N. R., Jr. 2001, ApJ, 562, 558

Kallenrode, M.-B., Cliver, E. W., \& Wibberenz, G. 1992, ApJ, 391, 370

Kanbach, G. O., et al. 1993, Aston. Astrophys. Suppl., 97, 349

Klecker, B., Hovestadt, D., et al. 1984, ApJ, 281, 458

Lange, I. \& Forbush, S. E. 1942a, Terr. Mag., 47, 185

Lange, I. \& Forbush, S. E. 1942b, Terr. Mag., 47, 331

Lee, M. A. 2005, ApJS, 158, 38

Lin, R. P. 1970, Solar Phys., 12, 266

Mason, G. M., Reames, D. V., et al. 1986, ApJ, 303, 849

Mason, G. M., et al. 2002, ApJ, 574, 1039

Mazur, J. E., Mason, G. M., et al. 1999, Geophys. Res. Lett., 26, 173

Meyer, P., Parker, E. N., \& Simpson, J. A. 1956, Phys. Rev., 104, 768

Moses, D., Dröge, W., Meyer, P., \& Evenson, P. 1989, ApJ, 346, 523

Nitta, N. V., Cliver, E. W., \& Tylka, A. J. 2003, ApJ (Lett.), 586, L103

Ramaty, R., Murphy, R. J., \& Dermer, C. D. 1987, ApJ (Lett.), 216, L41

Reames, D. V., von Rosenvinge, T. T., \& Lin, R. P. 1985, ApJ, 292, 716

Reames, D. V., Stone, R. G., \& Kallenrode, M.-B. 1991, ApJ, 380, 287

Reames, D. V. 1993, Adv. Sp. Res., 13(9), 331

Reames, D. V. 1999, Space Sci. Revs, 90, 413

Reid, G. C. 1964, J. Geophys. Res., 69, 2659

Ryan, J. M. 2000, Space Sci. Revs, 93, 581 
Tsurutani, B. T., and Lin, R. P. 1985, J. Geophys. Res., 90, 1

Tylka, A. J., et al. 2005, ApJ, 625, 474

Tylka, A. J., et al. 2006, ApJS, 164, 536

Tylka, A. J. \& Lee, M. A. 2006, ApJ, 646, 1319

Wild, J. P., Smerd, S. F., \& Weiss, A. A. 1963, ARAA, 1, 291

Zhang, J., Dere, K. P., Howard, R. A., Kundu, M. R., \& White, S. M. 2001, ApJ, 561, 396

\section{Discussion}

SPANGLER: One would think that a CME would generate a quasi-parallel shock (if it expands radially). Are the quasi-perpendicular shocks you mention attributed to fast, transverse expansion of the CME initiation?

Cliver: Yes, such lateral expansion was observed in the LASCO C1 coronagraph.

FISK: Recent works on suprathermal tails in the solar wind have shown that the tails are enriched in ${ }^{3} \mathrm{He}+\mathrm{Fe}$. How would your paradigm change if this is a seed population for enhanced ${ }^{3} \mathrm{He}+\mathrm{Fe}$ in CME-driving solar flare events?

ClIVER: It may improve matters as there is some question that there are sufficient flare seed particles to account for the quasi-perpendicular events.

MeLnikOv: On your last figure you showed a nice correlation between fluxes of mildly relativistic electrons and energetic protons. This means that both populations are accelerated in the same process, and opens an opportunity for diagnostics of proton fluxes using the radio emission generated by those electrons in the corona. For example decameter radio emission would be a good indicator of such particle acceleration and propagation through the high corona. Do you know some studies about this kind of radio diagnostic?

Cliver: Cane et al. (2002) considered the decametric range. But the emission in this frequency range is from decidedly much lower energy electrons.

MACDOWALL: Is it correct that you have not used metric-decametric radio data or 1-10 MHZ type III burst complexity when identifying type III events for your 2009 paper?

Cliver: Yes, in part because there is at present no objective definition of a complex type III burst. Even then I would expect such bursts to be highly associated with DH type II bursts. 